\title{
CORRESPONDENCE
}

\author{
(To the Editors of the Journal of the Institute of Actuaries)
}

\section{Mortality of European members of the Services in India}

SIRS,

In the discussion following the reading of Professor Vaidyanathan's paper on Mortality of Indian Assured Lives (F.I.A. Vol. Lxx, p. I5), Mr D. C. Fraser remarked that it would be of interest and value if the results of the successive investigations which had been made into the mortality of the members of the Indian Civil Service could be recorded.

The investigations referred to by Mr Fraser were made in connexion with the periodical valuations of the Indian Civil Service Family Pcnsion Fund, which was established in $188 \mathrm{I}$ for the benefit of the widows and orphans of members of the Indian Civil Service. A similar scheme, the Indian Military Service Family Pension Fund, was started in 1873 for the provision of pensions for the widows and orphans of military, medical, naval and ecclesiastical officers in the service of the Government of India. This scheme was closed at the end of 1914 to new entrants, for whom the Indian Military Widows' and Orphans' Fund was then established. All new entrants into the respective services are required to contribute under the appropriate scheme, membership of which is compulsory.

In the Indian Civil Service scheme, certain of the pensions are supplemented by payments from the Government, but apart from these additions, the Fund is self-supporting and is maintained by contributions paid by the members.

The military schemes are entirely self-supporting and normally involve no charge on Government funds. The benefits, which are smaller than those of the civil scheme, are additional to those granted by Royal Warrant from Indian revenues to the widows and families of military officers in the service of the Government of India.

In I928 a fourth Fund, the Superior Services (India) Family Pension Fund, was instituted to secure suitable provision for the widows and orphans of European and Anglo-Indian members of certain Civil Services other than the Indian Civil Service. This Fund, entry into which is now confined to officers appointed to the Indian Police, is also self-supporting. Its provisions are on much simpler lines than those of the older schemes which are of great complexity, involving many interesting actuarial problems. In view of its recent establishment, the experience of the Superior Services scheme so far available is limited, and the Fund is mentioned only to avoid any misconception regarding the constitution of the membership of the Indian Civil Service Family Pension Fund.

Following the discussion referred to above, inquiries had been received from various quarters regarding the mortality experience of the members of the Indian Services. Through the courtesy, which actuaries will desire to acknowledge, of the Secretary of State for India, it is possible for details to be made available to the actuarial profession of the mortality experience of the members of the schemes.

Tables are accordingly enclosed showing the experience in the three quinquennial periods of the members of (I) the Indian Civil Service scheme between I April I918 and 31 March 1933, and (2) the combined military schemes between I April r919 and 31 March 1934. 


\section{Table I. Indian Civil Service Family Pension Fund}

Mortality experience of European officers between I April I9I8 and 31 March 1937

Expected deaths on basis of Government Life Annuitants, I900-20 (Males) ultimate table

\begin{tabular}{|c|c|c|c|c|c|c|c|c|c|c|}
\hline \multicolumn{3}{|c|}{ Age group } & \multirow[t]{2}{*}{$\begin{array}{l}\text { Exposed } \\
\text { to risk }\end{array}$} & $\begin{array}{l}\text { Actual } \\
\text { deaths }\end{array}$ & \multirow[t]{2}{*}{$\begin{array}{l}\text { Expected } \\
\text { deaths }\end{array}$} & \multirow[t]{2}{*}{$\begin{array}{c}\text { Ratio } \\
\text { Actual/ } \\
\text { Expected }\end{array}$} & \multirow[t]{2}{*}{$\begin{array}{l}\text { Exposed } \\
\text { to risk }\end{array}$} & \multirow{2}{*}{$\underbrace{\begin{array}{c}\text { Actual } \\
\text { deaths }\end{array}}_{1023}$} & \multirow{2}{*}{$\begin{array}{c}\text { Expected } \\
\text { deaths }\end{array}$} & \multirow[t]{2}{*}{$\begin{array}{c}\text { Ratio } \\
\text { Actual/ } \\
\text { Expected }\end{array}$} \\
\hline & & & & $1918-23$ & & & & & & \\
\hline $\begin{array}{l}25 \\
30 \\
35 \\
40\end{array}$ & $\begin{array}{l}\text { Under } 25 \\
\& \text { under } \\
", \quad \text { " } \\
", \\
" \quad \text { " }\end{array}$ & $\begin{array}{l}30 \\
35 \\
40 \\
45\end{array}$ & $\begin{array}{r}30 \\
57 x \\
1083 \\
1155 \\
1098\end{array}$ & $\begin{array}{r}- \\
3 \\
7 \\
6 \\
10\end{array}$ & $\begin{array}{r}\cdot 1 \\
2 \cdot 5 \\
5 \cdot 3 \\
6 \cdot 8 \\
7 \cdot 9\end{array}$ & $\begin{array}{r}\cdot 00 \\
x \cdot 20 \\
x \cdot 32 \\
\cdot 88 \\
x \cdot 27\end{array}$ & $\begin{array}{r}61 \\
382 \\
700 \\
1056 \\
\text { I101 }\end{array}$ & $\begin{array}{l}1 \\
2 \\
5 \\
6 \\
5\end{array}$ & $\begin{array}{r}3 \\
I \cdot 6 \\
3 \cdot 5 \\
6 \cdot 4 \\
7 \cdot 8\end{array}$ & $\begin{array}{r}3 * 33 \\
I * 25 \\
I * 43 \\
\cdot 94 \\
\cdot 64\end{array}$ \\
\hline \multicolumn{3}{|c|}{ Under 45} & 3937 & $26 *$ & $22 \cdot 6$ & $I \cdot T 5$ & 3300 & 19 & $19 \cdot 6$ & .97 \\
\hline $\begin{array}{l}45 \\
5 \circ \\
55\end{array}$ & $\begin{array}{c}\& \text { under } \\
", " \text { " }\end{array}$ & $\begin{array}{l}50 \\
55 \\
60\end{array}$ & $\begin{array}{r}r 49 I \\
984 \\
485\end{array}$ & $\begin{array}{r}12 \\
12 \\
3\end{array}$ & $\begin{array}{r}I 3 \cdot 0 \\
I I \cdot I \\
7 \cdot 8\end{array}$ & $\begin{array}{r}\cdot 92 \\
\mathbf{1}+08 \\
\cdot 38\end{array}$ & $\begin{array}{r}I 053 \\
I 439 \\
924\end{array}$ & $\begin{array}{r}5 \\
8 \\
17\end{array}$ & $\begin{array}{r}9.2 \\
16.7 \\
15.0\end{array}$ & $\begin{array}{r}\cdot 54 \\
-48 \\
I \cdot 13\end{array}$ \\
\hline 45 & " , " & 60 & 2960 & 27 & 359 & $\cdot 85$ & 3410 & 30 & $40 \cdot 9$ & -73 \\
\hline $\begin{array}{l}60 \\
65 \\
70 \\
75\end{array}$ & $\begin{array}{cc}\text { " } & \text { " } \\
\text { " } & \text { "' } \\
\text { \& } & \text { over }\end{array}$ & $\begin{array}{l}65 \\
70 \\
75\end{array}$ & ${ }^{6 \mathrm{I}}$ & $\frac{2}{-}$ & $\begin{array}{l}I \cdot 4 \\
- \\
-\end{array}$ & $\frac{1443}{-}$ & $\begin{array}{r}450 \\
50 \\
- \\
-\end{array}$ & $\begin{array}{r}9 \\
2 \\
-\end{array}$ & $\begin{array}{r}\text { I I.O } \\
\text { I.8 } \\
- \\
-\end{array}$ & $\begin{array}{r}.82 \\
1 \cdot 11 \\
- \\
\end{array}$ \\
\hline 60 & $\&$ over & & $6 \mathrm{I}$ & 2 & $I \cdot 4$ & $I \cdot 43$ & 500 & II & $12 \cdot 8$ & $\cdot 86$ \\
\hline \multicolumn{3}{|c|}{ Total } & 6958 & $55^{*}$ & 55.9 & $\cdot 98$ & 7216 & 60 & $73 \cdot 3$ & .82 \\
\hline $\begin{array}{l}25 \\
30 \\
35 \\
40\end{array}$ & $\begin{array}{c}\text { Under } 25 \\
\text { \& under } \\
", \quad \text { ", } \\
", \quad "\end{array}$ & $\begin{array}{l}30 \\
35 \\
40 \\
45 \\
\end{array}$ & $\begin{array}{r}181 \\
495 \\
374 \\
672 \\
1002\end{array}$ & $\frac{1}{3}$ & $\begin{array}{r}8-33 \\
\cdot 7 \\
2 \cdot 0 \\
1 \cdot 9 \\
4 \cdot 1 \\
7 \cdot 1\end{array}$ & $\begin{array}{r}I \cdot 43 \\
I \cdot 50 \\
\cdot 00 \\
\cdot 98 \\
\cdot 85\end{array}$ & $\begin{array}{r}74 \\
548 \\
324 \\
320 \\
549\end{array}$ & $\begin{array}{l}1933 \\
- \\
3 \\
I \\
2 \\
3\end{array}$ & $\begin{array}{r}* 3 \\
2 * 4 \\
1 \cdot 7 \\
I \cdot 9 \\
3 \cdot 9\end{array}$ & $\begin{array}{r}\cdot 00 \\
I \cdot 25 \\
\cdot 59 \\
I \cdot 05 \\
.77\end{array}$ \\
\hline \multicolumn{3}{|c|}{ Under 45} & 2724 & 14 & I $5 \cdot 8$ & .89 & I8I5 & 9 & 10.2 & .88 \\
\hline $\begin{array}{l}45 \\
50 \\
55\end{array}$ & $\begin{array}{cc}\& & \text { under } \\
" & " \\
" & " 1\end{array}$ & & $\begin{array}{l}1050 \\
1013 \\
1328\end{array}$ & $\begin{array}{r}7 \\
9 \\
37\end{array}$ & $\begin{array}{r}9 \cdot 2 \\
11 \cdot 8 \\
22 \cdot 1\end{array}$ & $\begin{array}{r}-76 \\
-76 \\
I \cdot 67\end{array}$ & $\begin{array}{l}784 \\
794 \\
755\end{array}$ & $\begin{array}{r}5 \\
6 \\
10\end{array}$ & $\begin{array}{r}7 \cdot 0 \\
9 \cdot 1 \\
12 \cdot 6\end{array}$ & $\begin{array}{l}.71 \\
.66 \\
.79\end{array}$ \\
\hline 45 & , , , & 60 & 3391 & 53 & $43 \cdot 1$ & $1 \cdot 23$ & 2333 & $2 I$ & $28 \cdot 7$ & 73 \\
\hline $\begin{array}{l}60 \\
65 \\
70 \\
75\end{array}$ & $\begin{array}{rr}" & " \\
" & " \\
\& & \text { over }\end{array}$ & $\begin{array}{l}65 \\
70 \\
75\end{array}$ & $\begin{array}{r}836 \\
394 \\
38 \\
\end{array}$ & $\begin{array}{r}I_{3} \\
\text { I5 } \\
2 \\
\end{array}$ & $\begin{array}{r}20 \cdot 7 \\
\text { I } 5 \cdot 5 \\
2 \cdot 1 \\
\end{array}$ & $\begin{array}{l}.63 \\
.97 \\
.95 \\
\end{array}$ & $\begin{array}{r}974 \\
539 \\
223 \\
10\end{array}$ & $\begin{array}{r}28 \\
20 \\
17 \\
1\end{array}$ & $\begin{array}{r}25 \cdot 0 \\
21 \cdot 6 \\
13 \cdot 6 \\
.9\end{array}$ & $\begin{array}{r}1 \cdot 12 \\
\cdot 93 \\
1 \cdot 25 \\
1 \cdot 11\end{array}$ \\
\hline 60 & $\&$ over & & $I 268$ & 30 & $3^{8 \cdot 3}$ & .78 & 1746 & 66 & $6 I \cdot I$ & 1.08 \\
\hline & Total & & 7383 & 97 & 972 & 100 & 5894 & 96 & 100.0 & .96 \\
\hline
\end{tabular}

* Excluding four deaths due to the 1914-18 war. 


\section{Table 2. Indian Military Service Family Pension Fund and Indian Military Widows' and Orphans' Fund}

Combined mortality experience of European officers between I April I919 and 31 March 1937

Expected deaths on basis of Government Life Annuitants, 1900-20 (Males) ultimate table

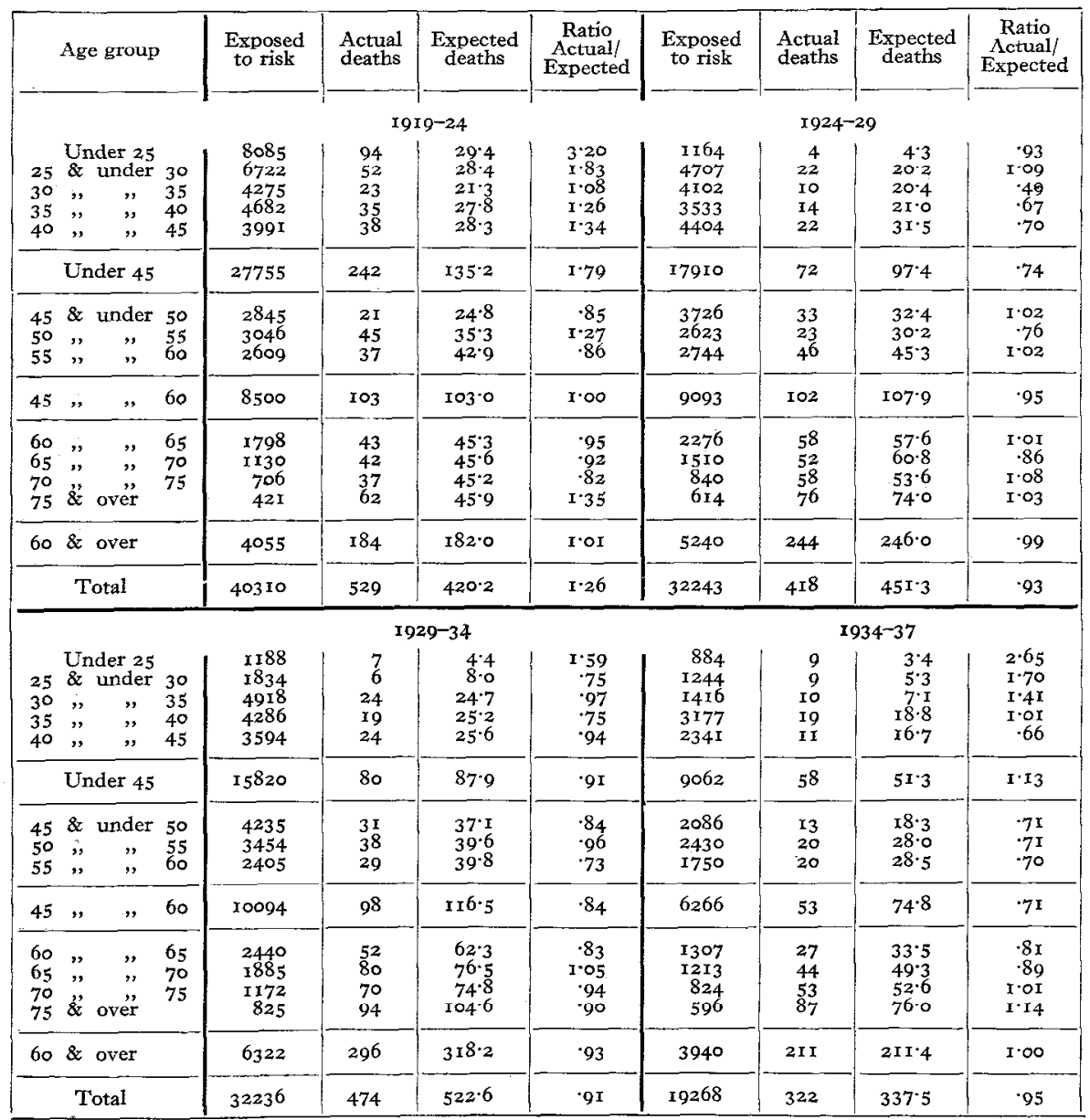

Note. The experience is subsequent to the $19 \mathrm{I} 4-18$ war, but in the period covered includes deaths from all causes. 


\section{Correspondence}

Valuations at 3I March 1937 of all the Funds were required for a special purpose; the tables also include the results of the respective experiences during the four years and three years since the preceding quinquennial valuations.

The tables show the exposed to risk and the deaths in five-yearly age groups, and also the expected deaths, calculated throughout on the basis of the Government Life Annuitants, 1900-20, table as a convenient standard for comparative purposes. The ratios have been inserted in all cases, although for some groups their significance is limited by the smallness of the numbers involved.

The figures relate to the members of the family pension schemes, and include both serving and retired officers. Subsidiary totals are given for three broad age groups :

(I) Under age 45 , consisting in the main of serving members.

(2) Age 45 to age 60 , the range within which retirement ordinarily takes place on completion of the normal full period of service.

(3) Age 6o and over, consisting almost entirely of retired members, since very few officers, civil or military, remain in service beyond age 60 .

I am, Sirs,

Your obedient Servant,

D. A. PORTEOUS

\section{Government Actuary's Department \\ Caxton House East \\ Tothill Street \\ London, S.W. I}

9 November 1944 